\title{
General Bounds on Electronic Shot Noise in the Absence of Currents
}

\author{
Jakob Eriksson, ${ }^{1,2}$ Matteo Acciai $\odot,{ }^{1}$ Ludovico Tesser, ${ }^{1}$ and Janine Splettstoesser ${ }^{1}$ \\ ${ }^{1}$ Department of Microtechnology and Nanoscience (MC2), Chalmers University of Technology, S-412 96 Göteborg, Sweden \\ ${ }^{2}$ University of Gothenburg, S-412 96 Göteborg, Sweden
}

(Received 2 March 2021; accepted 12 August 2021; published 21 September 2021)

\begin{abstract}
We investigate the charge and heat electronic noise in a generic two-terminal mesoscopic conductor in the absence of the corresponding charge and heat currents. Despite these currents being zero, shot noise is generated in the system. We show that, irrespective of the conductor's details and the specific nonequilibrium conditions, the charge shot noise never exceeds its thermal counterpart, thus establishing a general bound. Such a bound does not exist in the case of heat noise, which reveals a fundamental difference between charge and heat transport under zero-current conditions.
\end{abstract}

DOI: 10.1103/PhysRevLett.127.136801

Introduction.-In mesoscopic devices, noise arises both at equilibrium, due to thermal excitations leading to thermal noise [1,2], and out of equilibrium, where partitioning of electrons at a scatterer generates shot noise [3]. Shot noise measurements have proven extremely useful to gain insights on charge carriers [4] and have led, for instance, to the experimental observation of fractional charges $[5,6]$. Very recently, a distinct type of shot noise has been measured: this so-called delta- $T$ noise is purely due to a temperature bias, but is distinct from thermal noise and is finite despite the average current vanishes [7-9]. This previously overlooked source of nonequilibrium noise was first introduced for diffusive conductors [10] and is currently attracting a lot of attention, both from a theoretical [11-13] and from an experimental perspective [7-9,14]. Until now, the analysis of this phenomenon has however mostly been restricted by the following constraints: (i) conductors are assumed to have energy-independent transmission probabilities, (ii) the nonequilibrium state is induced by a pure temperature bias, and (iii) only chargecurrent shot noise has been considered.

In this Letter, we generalize this broadly by lifting all three constraints and thereby provide concrete results for a wider class of noise phenomena, of which the delta- $T$ noise is a specific manifestation. Allowing the conductor to have an energy-dependent transmission probability, we open up the analysis for two important aspects. Finite shot noise arises at zero-average current under generic nonequilibrium conditions, e.g., combinations of temperature and voltage biases important for thermoelectrics. Moreover, not only

Published by the American Physical Society under the terms of the Creative Commons Attribution 4.0 International license. Further distribution of this work must maintain attribution to the author(s) and the published article's title, journal citation, and DOI. Funded by Bibsam. the charge, but also other types of currents are subject to shot noise persisting at zero current under appropriate nonequilibrium conditions: as an example, we study the heat shot noise in the absence of an average heat current. Importantly, we find a fundamental bound for the charge shot noise under the condition of zero current: it can never exceed the thermal noise, independently of the system details and the type of nonequilibrium conditions. In contrast, such a bound does not exist for the heat shot noise in the absence of a heat current.

The presented analysis of zero-current charge and heat shot noise will also be particularly relevant for the topical field of quantum thermodynamics [15-17]. In nonequilibrium thermal engines, average currents between source

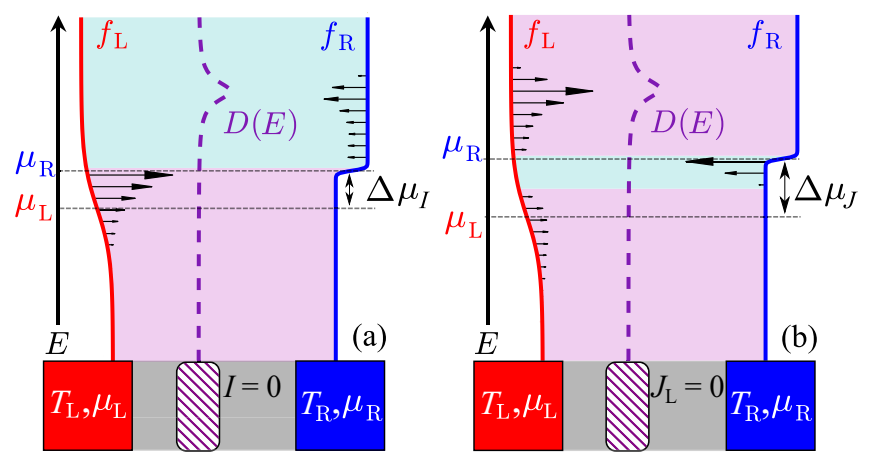

FIG. 1. Illustration of nonequilibrium conditions leading to vanishing charge (a) and heat (b) current into contact $L$ of the setup sketched at the bottom. Fermi functions $f_{L}$ and $f_{R}$ of left and right contact with $T_{L}>T_{R}$ and with $\mu_{L}$ and $\mu_{R}$ are shown as red and blue lines. The purple dashed line shows the example of a Lorentzian transmission probability of the scatterer inside the conductor (gray). In order to fulfill the zero-current condition, we have (a) $\mu_{R}-\mu_{L}=\Delta \mu_{I}$ and (b) $\mu_{R}-\mu_{L}=\Delta \mu_{J}$. Arrows show the magnitude and direction (emphasized by the background color) of the resulting energy-resolved fluxes adding up to zeroaverage charge or heat current. 
and working substance can vanish [18-21], while their noise remains relevant [22-24]. Also the minimization of noise as a performance goal of small-scale heat engines has recently been highlighted [25-28], and correlations of heat and charge currents have been linked to their efficiency $[29,30]$.

Here, we study a generic two-terminal conductor as sketched at the bottom of Fig. 1, using scattering theory. The energy dependence of the conductor's transmission probability goes along with a combination of $\Delta \mu$ and $\Delta T$ fulfilling the requirement of a vanishing current. Considering a temperature bias $\Delta T$, the voltage required for zero charge current is the thermovoltage $\Delta \mu=\Delta \mu_{I}$, which naturally develops in a thermoelectric system under open-circuit conditions. Alternatively, it is possible to completely suppress the heat current into one of the terminals, despite heat being generally generated at nonequilibrium. For a given temperature bias, this suppression always requires the presence of a nonvanishing heat thermovoltage $\Delta \mu_{J}$. In both situations, we find the corresponding shot noise to persist; we refer to this as the zerocurrent shot noise in the following. This zero-current shot noise arises because opposite energy-resolved currents are present in the system (see Fig. 1). We find analytical expressions for the zero-current charge and heat shot noise that can maximally be reached in conductors with constant transmission, requiring large temperature bias. Importantly, these limits can be exceeded, when the transmission is energy-dependent. Indeed, the zero-current charge shot noise can approach the corresponding thermal noise, and the heat shot noise is not bounded at all.

Formalism and model.-We consider a quantum conductor connected to two reservoirs $(\alpha=L, R)$ characterized by Fermi distributions $f_{\alpha}(E)=\left\{1+\exp \left[\beta_{\alpha}\left(E-\mu_{\alpha}\right)\right]\right\}^{-1}$, where $\mu_{\alpha}$ are the chemical potentials and $\beta_{\alpha}=\left(k_{B} T_{\alpha}\right)^{-1}$ the inverse temperatures. In the following, we fix $\mu_{L} \equiv 0$. We use the framework of scattering theory $[4,31]$, where the conductor is described by a transmission probability $D(E)$ that an electron at energy $E$ is transmitted from one reservoir to the other. This description is valid for conductors with negligible or weak electron-electron interactions that can be treated at mean-field level. In the recent measurements of delta- $T$ noise, scattering theory showed a very good agreement with experimental data in molecular junctions [7], quantum point contacts [8], and tunnel junctions [9]. In the following, we investigate the more general situation of zero-current charge and heat shot noise. Thus, we require vanishing charge current between the contacts, and heat current into contact $\alpha$. These are found from the expectation values of the operators $\hat{X}=\hat{I}_{L}=-\hat{I}_{R} \equiv \hat{I}$ and $\hat{X}=\hat{J}_{\alpha}=\hat{I}_{\alpha}^{E}-\mu_{\alpha} \hat{I}_{\alpha}$, and they are

$$
X=\frac{2}{h} \int d \operatorname{ExD}(E)\left[f_{L}(E)-f_{R}(E)\right],
$$

with $x \rightarrow\left\{-e, E-\mu_{\alpha}\right\}$ for $X \rightarrow\left\{I, J_{\alpha}\right\}$ and the elementary charge $e>0$. The zero-frequency noise of these currents is defined as $S^{X}=2 \int\langle\delta \hat{X}(t) \delta \hat{X}(0)\rangle d t$, where $\delta \hat{X}=\hat{X}-X$ is the fluctuation of the operator $\hat{X}$ around its average value $X$. The noise can be divided into two contributions $S^{X}=S_{\mathrm{th}}^{X}+S_{\mathrm{sh}}^{X}$,

$S_{\mathrm{th}}^{X}=\int d E \frac{4 x^{2}}{h} D(E) \sum_{\alpha=L, R} f_{\alpha}(E)\left[1-f_{\alpha}(E)\right]$,

$S_{\mathrm{sh}}^{X}=\int d E \frac{4 x^{2}}{h} D(E)[1-D(E)]\left[f_{L}(E)-f_{R}(E)\right]^{2}$.

The first term $S_{\mathrm{th}}^{X}$ is thermal-like noise. It contains independent contributions from the two contacts $\alpha=L, R$ and is therefore present even at equilibrium. The charge-current noise at equilibrium is the famous Johnson-Nyquist noise $[1,2]$. In contrast, the shot noise term $S_{\mathrm{sh}}^{X}$ is only present out of equilibrium, when $f_{L} \neq f_{R}$, and it is usually associated with the partitioning of a current flowing through the system. The standard situation is indeed when a pure voltage bias $\Delta \mu=\mu_{L}-\mu_{R}$ is applied, resulting in a net current $I \neq 0$. Then $S_{\mathrm{sh}}^{I}$ reduces to conventional shot noise [3]. However, as is clear from Eqs. (1) and (2b), a finite shot noise does not require a finite average current, $I \neq 0$. Indeed, another possibility to obtain a measurable shot noise, largely unexplored until recently, is to impose a pure temperature bias $\Delta T=T_{L}-T_{R}$. Then, it has been found for energyindependent transmission probabilities that $S_{\mathrm{sh}}^{I} \neq 0$ even though $I=0$. This is the recently investigated delta$T$ noise [7-9].

General bounds on charge and heat shot noise.-We start by providing general bounds on the charge-current noise in the absence of a charge current for a generic twoterminal system, described by a transmission probability $D(E)$, and with an arbitrary voltage and temperature bias. By solving a variational problem following the method used in Ref. [32], one can show that the uniform transmission probability $D(E)=1 / 2$ maximizes the charge shot noise also under the condition of vanishing current [33]. This uniform transmission leads at the same time to a possibly large thermal noise. Indeed, we find that for any transmission probability, the zero-current charge shot noise is bounded by the thermal noise. The inequalities

$$
S_{\mathrm{sh}}^{I} \leq \frac{4 e^{2}}{h} \int d E D(E) f_{L}(E)\left[1-f_{R}(E)\right] \leq S_{\mathrm{th}}^{I}
$$

can be obtained using only that $0 \leq\left\{D(E), f_{\alpha}(E)\right\} \leq 1$, the monotonicity of $f_{\alpha}(E)$ and the zero-current condition. Note that this inequality can be also extended to a system with many conduction channels and can hence even be applied to other types of conductors such as normalsuperconducting junctions [33]. Hence, the charge shot 
noise can never be greater than the thermal noise, fixing the general bound

$$
R_{I} \equiv\left[S_{\mathrm{sh}}^{I} / S_{\mathrm{th}}^{I}\right]_{I=0} \leq 1 .
$$

Interestingly, at large temperature bias and with a small and sharp transmission probability, it is possible to approach equality in Eq. (4). Concretely, this requires a transmission that is nonzero within an energy interval $\delta$ satisfying $\max \left(T_{L}, T_{R}\right) \gg \delta / k_{B} \gg \min \left(T_{L}, T_{R}\right)$ and $D(E) \ll 1$. To show that such a transmission optimizes the noise ratio $R_{I}$, we assume without loss of generality that $T_{L} \gg T_{R}$. Then, the mentioned conditions allow us to approximate the shot noise $\left(h / 4 e^{2}\right) S_{\mathrm{sh}}^{I}$ as

$$
D^{+} f_{L}^{2}\left(\epsilon_{0}\right)\left(\epsilon_{0}+\frac{\delta}{2}-\mu_{R}\right)+D^{-}\left[1-f_{L}\left(\epsilon_{0}\right)\right]^{2}\left(\mu_{R}+\frac{\delta}{2}-\epsilon_{0}\right) .
$$

Here, we call $\epsilon_{0}$ the energy around which $D(E) \neq 0$, and $D^{ \pm}$is the integral mean value of $D(E)$ for $E \gtrless \mu_{R}$ : $D^{ \pm}=\left(\epsilon_{0}-\mu_{R} \pm \delta / 2\right)^{-1} \int_{\mu_{R}}^{\epsilon_{0} \pm \delta / 2} D(E) d E$. The zero-current condition fixes $\mu_{R}$ such that we obtain

$$
S_{\mathrm{sh}}^{I} \approx \frac{4 e^{2}}{h} \frac{\delta f_{L}\left(\epsilon_{0}\right)\left(1-f_{L}\left(\epsilon_{0}\right)\right) D^{+} D^{-}}{D^{-}\left[1-f_{L}\left(\epsilon_{0}\right)\right]+D^{+} f_{L}\left(\epsilon_{0}\right)} \approx S_{\mathrm{th}}^{I},
$$

showing that indeed $R_{I} \rightarrow 1$. An example where this occurs is the Lorentzian function introduced in Eq. (8).

In contrast, the zero-current heat shot noise is not bounded by the heat thermal noise, thus revealing a profound difference between charge transport and heat or energy transport. Essentially, this is due to the larger contribution to heat transport of electrons with higher energies. Choosing a transmission that allows for transport in two separate energy regions and increasing the separation distance between them, the heat shot noise can become arbitrarily large [33], still maintaining a zero heat current and a limited thermal noise. This does not apply to charge transport due to the electrons' fixed charge, thus limiting the magnitude of zero-current charge shot noise.

Charge-current noise.-We now move on to show explicit results for the zero-current shot noise. First, we notice that if the temperatures satisfy $\Delta T \ll \bar{T} \equiv$ $\left(T_{L}+T_{R}\right) / 2$, the shot noise is very small compared with the thermal one (see for instance Ref. [7]) both for charge and heat. Therefore, we focus henceforth on a large temperature bias, where the shot noise contribution can be significant. For a generic, weakly energy-dependent transmission function, we find for the ratio between the two noise contributions:

$$
R_{I}=[1-D(0)]\left[\ln \left(2+2 \cosh \frac{\Delta \mu_{I}}{k_{B} T_{L}}\right)-1\right] .
$$

Here, $\beta_{L} \Delta \mu_{I} \propto D^{\prime}(0) k_{B} T_{L} / \gamma$ [33] and $\gamma$ indicates a typical energy scale set by $D(E)$, while $D\left(E=\mu_{L}\right)=D(0)$ is the transmission in the vicinity of the transport window. This generalizes a previous result for delta- $T$ noise [9] to arbitrary, weakly energy-dependent transmission probabilities, requiring only $\gamma \gg k_{B} T_{L}$. For a constant transmission, $D(E)=D^{0}$, the thermovoltage is $\Delta \mu_{I}=0$ (as no thermoelectric effects arise in this particle-hole symmetric case), and Eq. (6) reduces to

$$
\left.R_{I}\right|_{D(E)=D^{0}}=\left(1-D^{0}\right)(2 \ln 2-1) .
$$

Interestingly, a direct numerical evaluation of Eq. 2 for arbitrary temperatures $T_{L}$ and $T_{R}$ [33] shows that Eq. (7) is an upper limit for $R_{I}$ in the case of a constant transmission. In contrast, we here find that a weak energy-dependence in $D(E)$ already breaks this limit. In the regime of validity of Eq. (6), the presence of the thermovoltage $\Delta \mu_{I} \neq 0$ always leads to an increase with respect to Eq. (7).

We now compare the above generic features of a weakly energy-dependent transmission with the results we obtain for the specific case of a Lorentzian

$$
D_{\text {Lor }}(E)=D^{0} \frac{\Gamma^{2}}{\Gamma^{2}+\left(E-\epsilon_{0}\right)^{2}},
$$

with arbitrarily sharp energy dependence as indicated by the purple dashed line in Fig 1. This experimentally relevant choice is of interest for highly efficient thermoelectrics [15,34-36] and is convenient for our purposes since it can model a broad range of conductor characteristics. A resonant level with a small width $\Gamma$ is an example of a transmission with which the charge shot noise approaches the thermal noise, when $k_{B} \max \left(T_{L}, T_{R}\right) \gg \Gamma \gg k_{B} \min \left(T_{L}, T_{R}\right)$. In contrast, large $\Gamma \gg k_{B} \max \left(T_{L}, T_{R}\right)$ reproduces the generic, weakly energy-dependent results of Eq. (6). The limit $\Gamma / k_{B} \max \left(T_{L}, T_{R}\right) \rightarrow \infty$, yielding a constant transmission, is the regime in which delta- $T$ noise was mainly studied previously; in the special case of $D^{0}=1 / 2$ the shot noise is maximized.

Using Eq. (8), we find $\Delta \mu_{I}$ by numerically solving the equation $I=0$. Plugging $\Delta \mu_{I}$ into Eq. (2) yields the resulting zero-current charge shot noise and thermal noise. The charge noise ratio $R_{I}$, normalized to $2 \ln 2-1$, together with the charge thermovoltage $\Delta \mu_{I}$ are shown in Fig. 2 as a function of the peak energy $\epsilon_{0}$ and the width $\Gamma$ of the Lorentzian, for $T_{L} \gg T_{R}$. Since $\Delta \mu_{I}\left(R_{I}\right)$ is an odd (even) function of $\epsilon_{0}$, we consider $\epsilon_{0} \geq 0$ only.

We find that the charge noise ratio approaches the value $R_{I}=2 \ln 2-1$ (green areas) in those parameter regimes where $D_{\text {Lor }}(E)$ is close to constant. This happens in the limit $\beta_{L} \Gamma \gg 1$, where $D_{\text {Lor }} \rightarrow D^{0}$, which we choose to be small compared with $1\left(D^{0}=10^{-2}\right.$, in Fig. 2). Likewise, when $\beta_{L} \epsilon_{0} \gg 1$, only the tail of the Lorentzian overlaps 


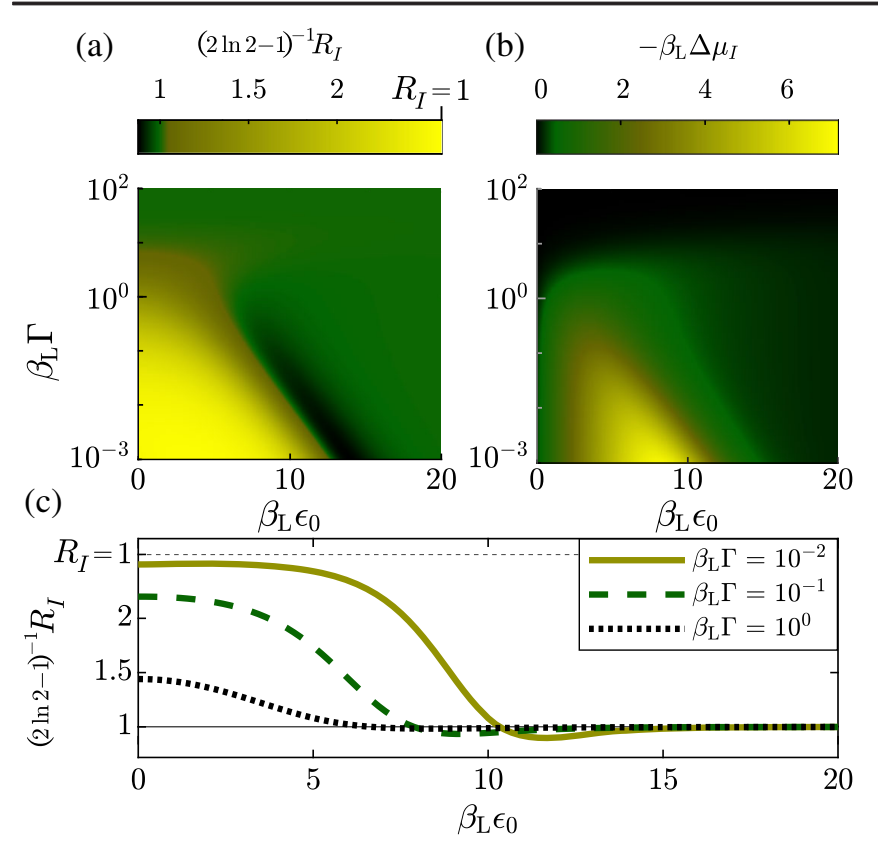

FIG. 2. (a) Charge noise ratio $R_{I}$, normalized to the characteristic factor $2 \ln 2-1$ [see Eq. (7)]. (b) Corresponding charge thermovoltage $\Delta \mu_{I}$. (c) Cuts of the density plot in (a) for different values of $\Gamma$. For all plots, we use $D(E)=D_{\text {Lor }}(E)$, with $D^{0}=10^{-2}$, and $T_{R} \ll T_{L}$ at fixed, finite $T_{L}$.

with the transport window [i.e., the region where the difference of Fermi functions in Eq. (1) is large], and the transmission function can be approximated by a constant much smaller than $D^{0}$. Correspondingly, we find $\Delta \mu_{I} \rightarrow 0$ in these regimes [see Fig. 2(b)].

Importantly, in those parameter regimes where the energy dependence of the transmission probability cannot be neglected, the charge noise ratio $R_{I}$ exceeds the value $2 \ln 2-1$ and approaches the fundamental bound $R_{I}=1$ [yellow regions in (b)]. This means that the zero-current charge shot noise can be equal to the thermal noise, as visible from Fig. 2(c), showing that a sharp, weakly transmitting Lorentzian is an example of the optimal functions discussed after Eq. (4). Interestingly, the limit $R_{I} \approx 1$ is not only reached at finite thermovoltages, but also for $\epsilon_{0} \rightarrow 0$, where $\Delta \mu_{I}=0$ due to electron-hole symmetry. We conclude that even the charge noise ratio $R_{I}$ for delta- $T$ noise, namely for zero-current charge shot noise at $\Delta \mu_{I}=0$, can be increased by more than a factor 2 compared with the case of constant transmission.

Heat current noise.-Importantly, the concept of zerocurrent shot noise is not limited to charge currents, but can greatly be extended to other transport quantities. Here, as an example, we show explicit results for the zero-current heat shot noise, which to our knowledge has not been studied before. This choice is motivated by the current interest in mesoscopic heat engines, where fluctuations in heat and power can play an important role [15]. Unlike conserved currents, the heat current in nonequilibrium conductors can in general be nullified only in one of the contacts at a time. We hence investigate the heat current noise in contact $L, S^{J_{L}} \equiv S^{J}$, at vanishing heat current into the same contact. This situation of nonvanishing zerocurrent heat shot noise can only be obtained when both a temperature and a voltage difference across the conductor are present.

Focusing again on the regime $T_{R} \ll T_{L}$ and considering first a generic, weakly energy-dependent transmission probability, with $k_{B} T_{L} \ll \gamma$, we find

$$
R_{J}=\frac{3}{\pi^{2}}[1-D(0)] A\left(x_{J}\right),
$$

where $x_{J}=\beta_{L} \Delta \mu_{J}$ is the dimensionless heat thermovoltage, $\quad A(x)=2 x^{2} \ln \left(1+e^{x}\right)-\left(\pi^{2}+x^{3}\right) / 3+$ $4 x \mathrm{Li}_{2}\left(-e^{x}\right)-4 \mathrm{Li}_{3}\left(-e^{x}\right)$, and $\mathrm{Li}_{n}$ the polylogarithm function. It is instructive to analyze Eq. (9) when $D(E)=D^{0}$. In this case, $x_{J}= \pm \pi / \sqrt{3}$ [37], and we find

$\left.R_{J}\right|_{D(E)=D^{0}}=\frac{3}{\pi^{2}} A\left(\frac{\pi}{\sqrt{3}}\right)\left(1-D^{0}\right) \equiv R_{J}^{0}\left(1-D^{0}\right)$.

Since $R_{J}^{0} \approx 0.45$, this shows that the zero-current heat shot noise can also be of the same order of magnitude as the corresponding thermal noise even for this simple transmission probability. As for the charge noise, we find that Eq. (10) provides an upper limit for $R_{J}$ when $D(E)=D^{0}$. This can already be exceeded for a weakly energydependent transmission, as $A\left(x_{J}\right)$ is an increasing function of $\left|x_{J}\right|$ and $x_{J} \propto \pm \pi / \sqrt{3}\left[1 \pm \zeta k_{B} T_{L} / \gamma\right]$, where $\zeta$ is a coefficient depending on the transmission [33].

We finally move to the Lorentzian-shaped transmission function, going beyond the limit of a weak energy dependence. By solving $J_{L}=0$, we find $\Delta \mu_{J}$, which we plug into Eq. (2) to obtain the zero-current heat shot noise and heat thermal noise; their ratio for $T_{R} \ll T_{L}$ is plotted in Fig. 3. The density plot for $R_{J}$, displayed in Fig. 3(a), shows extended regions (green) where the value $R_{J}=R_{J}^{0}$ is approached. As for the charge noise ratio, this occurs when $D(E)$ is close to constant and the heat thermovoltage [Fig. 3(b)] approaches $-\beta_{L} \Delta \mu_{J}=\pi / \sqrt{3}$. Interestingly, Fig. 3(a) shows that $S_{\text {sh }}^{J}$ does not exceed $S_{\text {th }}^{J}$ even though the heat shot noise is generally unbounded. The reason for this is that the Lorentzian transmission probability allows transport of particles in one connected energy interval only. Furthermore, Fig. 3 shows that, compared with $R_{I}$, there are more extended regions where $R_{J}<R_{J}^{0}$.

Conclusions. - This work greatly extends previous studies on delta- $T$ noise to more general nonequilibrium conditions and to different types of transport quantities. We have proven a universal bound, namely that the zerocurrent charge shot noise can never exceed the zero-current thermal charge noise. For heat noise, such a bound does not exist. 


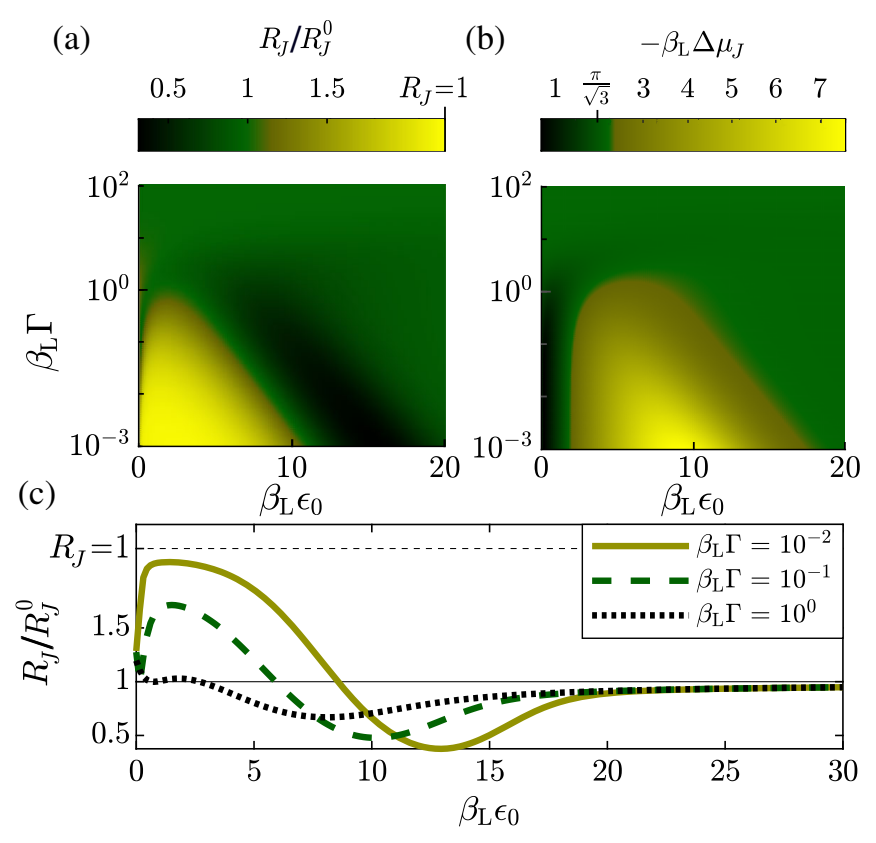

FIG. 3. (a) Ratio $R_{J}$ between the zero-current heat shot noise and heat thermal noise [normalized to the characteristic factor $R_{J}^{0}$, see Eq. (10)]. (b) Corresponding heat thermovoltage. (c) Cuts of the density plot in (a) for different values of $\Gamma$. For all plots, we use $D(E)=D_{\text {Lor }}(E)$, with $D^{0}=10^{-2}$, and $T_{R} \ll T_{L}$ at fixed, finite $T_{L}$.

While we have here focused on charge and heat transport, it would be interesting to investigate this concept for other kinds of currents such as spin currents and their noise in the future. Moreover, an analysis of out-of-equilibrium fluctuation relations at vanishing average current [38-40] would be of interest for conductors studied here, not fulfilling local detailed balance conditions [38,39]. Finally, the possible use of delta- $T$ noise for shot noise spectroscopy of thermal gradients has been pointed out [7], and we expect this scope to become broader with extensions to different types of shot noise under general nonequilibrium conditions as presented in this Letter.

We thank Rafael Sánchez, Jens Schulenborg, Christian Spånslätt, and Robert Whitney for helpful comments on the manuscript. We acknowledge financial support from the Swedish VR (J.S.) and the Knut and Alice Wallenberg Foundation (M. A., J. S., and L. T.).

[1] J. B. Johnson, Thermal agitation of electricity in conductors, Nature (London) 119, 50 (1927).

[2] H. Nyquist, Thermal agitation of electric charge in conductors, Phys. Rev. 32, 110 (1928).

[3] W. Schottky, Über spontane Stromschwankungen in verschiedenen Elektrizitätsleitern, Ann. Phys. (Berlin) 362, 541 (1918).

[4] Ya. M. Blanter and M. Büttiker, Shot noise in mesoscopic conductors, Phys. Rep. 336, 1 (2000).
[5] R. de Picciotto, M. Reznikov, M. Heiblum, V. Umansky, G. Bunin, and D. Mahalu, Direct observation of a fractional charge, Nature (London) 389, 162 (1997).

[6] L. Saminadayar, D. C. Glattli, Y. Jin, and B. Etienne, Observation of the $e / 3$ Fractionally Charged Laughlin Quasiparticle, Phys. Rev. Lett. 79, 2526 (1997).

[7] O. S. Lumbroso, L. Simine, A. Nitzan, D. Segal, and O. Tal, Electronic noise due to temperature differences in atomicscale junctions, Nature (London) 562, 240 (2018).

[8] E. Sivre, H. Duprez, A. Anthore, A. Aassime, F. D. Parmentier, A. Cavanna, A. Ouerghi, U. Gennser, and F. Pierre, Electronic heat flow and thermal shot noise in quantum circuits, Nat. Commun. 10, 5638 (2019).

[9] S. Larocque, E. Pinsolle, C. Lupien, and B. Reulet, Shot Noise of a Temperature-Biased Tunnel Junction, Phys. Rev. Lett. 125, 106801 (2020).

[10] E. V. Sukhorukov and D. Loss, Noise in multiterminal diffusive conductors: Universality, nonlocality, and exchange effects, Phys. Rev. B 59, 13054 (1999).

[11] E. Zhitlukhina, M. Belogolovskii, and P. Seidel, Electronic noise generated by a temperature gradient across a hybrid normal metal-superconductor nanojunction, Appl. Nanosci. 10, 5121 (2020).

[12] J. Rech, T. Jonckheere, B. Grémaud, and T. Martin, Negative Delta- $T$ Noise in the Fractional Quantum Hall Effect, Phys. Rev. Lett. 125, 086801 (2020).

[13] M. Hasegawa and K. Saito, Delta-T noise in the Kondo regime, Phys. Rev. B 103, 045409 (2021).

[14] R. A. Melcer, B. Dutta, C. Spånslätt, Jinhong Park, A. D. Mirlin, V. Umansky, Absent thermal equilibration on fractional quantum Hall edges over macroscopic scale, arXiv:2106.12486.

[15] G. Benenti, G. Casati, K. Saito, and R. S. Whitney, Fundamental aspects of steady-state conversion of heat to work at the nanoscale, Phys. Rep. 694, 1 (2017).

[16] R. S. Whitney, R. Sánchez, and J. Splettstoesser, Quantum thermodynamics of nanoscale thermoelectrics and electronic devices, in Thermodynamics in the Quantum Regime: Fundamental Aspects and New Directions, edited by F. Binder, L. A. Correa, C. Gogolin, J. Anders, and G. Adesso (Springer, Cham, 2018), pp. 175-206.

[17] F. Binder, L. A. Correa, C. Gogolin, J. Anders, and G. Adesso, Thermodynamics in the Quantum Regime (Springer International Publishing, Cham, Switzerland, 2018).

[18] R. Sánchez, J. Splettstoesser, and R. S. Whitney, Nonequilibrium System as a Demon, Phys. Rev. Lett. 123, 216801 (2019).

[19] S. E. Deghi and R. A. Bustos-Marún, Entropy current and efficiency of quantum machines driven by nonequilibrium incoherent reservoirs, Phys. Rev. B 102, 045415 (2020).

[20] F. Hajiloo, R. Sánchez, R. S. Whitney, and J. Splettstoesser, Quantifying nonequilibrium thermodynamic operations in a multiterminal mesoscopic system, Phys. Rev. B 102, 155405 (2020).

[21] S. Ciliberto, Autonomous out-of-equilibrium Maxwell's demon for controlling the energy fluxes produced by thermal fluctuations, Phys. Rev. E 102, 050103(R) (2020).

[22] R. Sánchez, B. Sothmann, A. N. Jordan, and M. Büttiker, Correlations of heat and charge currents in 
quantum-dot thermoelectric engines, New J. Phys. 15, 125001 (2013).

[23] N. Freitas and M. Esposito, Characterizing autonomous Maxwell demons, Phys. Rev. E 103, 032118 (2021).

[24] G. Marchegiani, A. Braggio, and F. Giazotto, Noise effects in the nonlinear thermoelectricity of a Josephson junction, Appl. Phys. Lett. 117, 212601 (2020).

[25] U. Seifert, Stochastic thermodynamics: From principles to the cost of precision, Physica (Amsterdam) 504A, 176 (2018).

[26] P. Pietzonka and U. Seifert, Universal Trade-Off between Power, Efficiency, and Constancy in Steady-State Heat Engines, Phys. Rev. Lett. 120, 190602 (2018).

[27] S. Kheradsoud, N. Dashti, M. Misiorny, P. P. Potts, J. Splettstoesser, and P. Samuelsson, Power, efficiency and fluctuations in a quantum point contact as steady-state thermoelectric heat engine, Entropy 21, 777 (2019).

[28] S. Pal, S. Saryal, D. Segal, T.S. Mahesh, and B. Kumar Agarwalla, Experimental study of the thermodynamic uncertainty relation, Phys. Rev. Research 2, 022044(R) (2020).

[29] A. Crépieux and F. Michelini, Mixed, charge and heat noises in thermoelectric nanosystems, J. Phys. Condens. Matter 27, 015302 (2015).

[30] P. Eyméoud and A. Crépieux, Mixed electrical-heat noise spectrum in a quantum dot, Phys. Rev. B 94, 205416 (2016).

[31] M. V. Moskalets, Scattering Matrix Approach to NonStationary Quantum Transport (World Scientific Publishing Company, London, England, UK, 2011).
[32] R. S. Whitney, Finding the quantum thermoelectric with maximal efficiency and minimal entropy production at given power output, Phys. Rev. B 91, 115425 (2015).

[33] See Supplemental Material at http://link.aps.org/supplemental/ 10.1103/PhysRevLett.127.136801 for further details on the derivation.

[34] L. D. Hicks and M. S. Dresselhaus, Effect of quantum-well structures on the thermoelectric figure of merit, Phys. Rev. B 47, 12727 (1993).

[35] G. D. Mahan and J. O. Sofo, The best thermoelectric, Proc. Natl. Acad. Sci. U.S.A. 93, 7436 (1996).

[36] M. Josefsson, A. Svilans, A. M. Burke, E. A. Hoffmann, S. Fahlvik, C. Thelander, M. Leijnse, and H. Linke, A quantum-dot heat engine operating close to the thermodynamic efficiency limits, Nat. Nanotechnol. 13, 920 (2018).

[37] This simple form is understood by equating the Joule heating $\Delta \mu_{J}^{2} / 2$ to the conduction part of the heat current $\pi^{2} k_{B}^{2} T_{L}^{2} / 6$.

[38] B. Altaner, M. Polettini, and M. Esposito, FluctuationDissipation Relations Far from Equilibrium, Phys. Rev. Lett. 117, 180601 (2016).

[39] L. Maisel and R. López, Effective equilibrium in out-ofequilibrium interacting coupled nanoconductors, Entropy 22, 8 (2019).

[40] I. Safi, Fluctuation-dissipation relations for strongly correlated out-of-equilibrium circuits, Phys. Rev. B 102, 041113(R) (2020). 